\title{
Pyomyositis mistaken for septic hip arthritis in children: the role of MRI in diagnosis and management
}

\author{
Franklin D. Shuler, MD, PhD'1, Grant Buchanan, MD'1, Cody Stover, MD'1, \\ Brock Johnson, MD', Milad Modarresi, MD' ${ }^{1}$, John Jasko, MD'
}

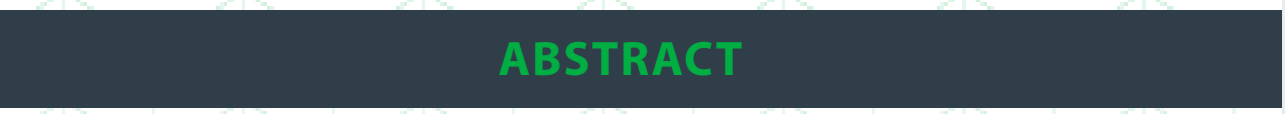

Septic arthritis is an orthopaedic emergency which requires timely management to prevent joint destruction and poor outcome. Differentiating septic arthritis from transient synovitis in pediatric patients is aided by the use of Kocher criteria which have excellent sensitivity but lack specificity. In addition to these two disorders, primary pyomyositis is bacterial infection of skeletal muscle that most commonly affects children. Patients present with pain, swelling, fever, and elevated inflammatory markers which mimic septic arthritis. If left untreated, pyomyositis can lead to abscess formation and sepsis. Due to potential for nearly identical presentations of septic arthritis and pyomyositis, differentiation of these two disorders can be aided with the use of MRI which has a high sensitivity for detecting muscle edema and abscess formation. In this case series, we discuss the use of MRI to assist with the diagnosis of pyomyositis versus septic arthritis. The authors advocate the use of MRI in questionable or complicated cases of septic arthritis or where synovial fluid aspiration is unable to be obtained promptly.

\section{KEYWORDS}

Pyomyositis, septic hip arthritis, MRI management

\section{INTRODUCTION}

Septic arthritis is an orthopaedic emergency which requires timely management to prevent joint destruction and poor outcome. The Kocher criteria are routinely used to distinguish septic arthritis from transient synovitis; these include inability to bear weight on the suspected extremity, fever, erythrocyte sedimentation rate (ESR) $>40 \mathrm{~mm} /$ $\mathrm{hr}$, and a peripheral white blood cell (WBC) count $>12,000$ cells $/ \mathrm{mm} 3 .{ }^{1}$ These criteria may be modified to incorporate C-reactive protein (CRP) with a level $>2.0 \mathrm{mg} / \mathrm{dl}$ suspect for active infection. ${ }^{2}$ Although these criteria may have excellent sensitivity, they lack specificity, leaving potential for misdiagnosis. This report presents two cases where pyomyositis was mistaken for septic arthritis of the hip. The purpose of this report is to demonstrate the indistinguishable presentation that pyomyositis and septic arthritis can produce in children, and highlight the value that MRI can have in the management of these patients.

\section{CASE HISTORY}

Patient 1

A 13 year old male with severe autism was transferred from an outside facility after caregivers noticed a two day history of refusal to bear weight on the right lower extremity (RLE). The patient was reportedly febrile prior to transfer, had a WBC count of 16,000 cells $/ \mathrm{mm} 3$ and an ESR of $37 \mathrm{~mm} / \mathrm{hr}$; he was given antipyretics as well as intravenous ceftriaxone and vancomycin outside of the hospital. Upon arrival, the patient was noted to have pain with manipulation of the right hip and his right lower extremity was warm to the touch. He was found to have a CRP of $10.4 \mathrm{mg} / \mathrm{dL}$, and a blood culture was positive for methicillin resistant staphylococcus aureus (MRSA). Due to the patient's severe autism and combative 
disposition, sedation was required to perform ultrasonography and aspiration of the hip. Given the signs of sepsis and high clinical suspicion for septic arthritis, the decision was made to proceed directly to hip arthrotomy, irrigation and debridement (I\&D), during which synovial samples were taken for culture and analysis. Synovial fluid analysis was negative for an infection and cultures demonstrated no growth. The patient underwent magnetic resonance imaging (MRI) of the pelvis and right lower extremity which revealed edema and abnormal enhancement of the vastus muscles and piriformis suggestive of diffuse pyomyositis (Figure 1). The patient subsequently underwent I\&D of the right thigh, where surgeons encountered a deep abscess and necrotic muscle around the vastus intermedius muscle. Intraoperative cultures were positive for MRSA. The patient was treated with intravenous vancomycin and underwent repeat I\&D on hospital days seven, eleven, and fourteen. He was discharged on hospital day twenty-six on vancomycin and had returned to his baseline status by the three week follow-up visit.

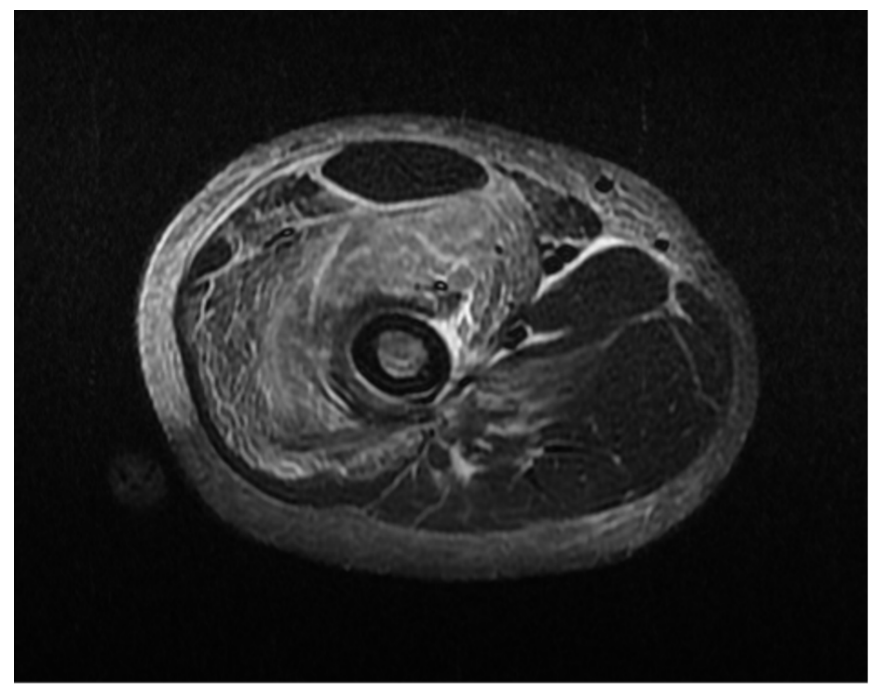

FIGURE 1: MRI of the right thigh of a 13-yearold male demonstrating edema and abnormal enhancement in the quadriceps muscles concerning for diffuse pyomyositis (axial STIR).

\section{Patient 2}

A 3 year old female presented to the emergency department complaining of a one day history of nausea, vomiting, right hip pain, and refusal to bear weight on the right lower extremity. According to parents, the patient had a fever the night before presentation; upon arrival she was afebrile. Physical examination revealed tenderness to palpation of the right hip and pain with right hip range of motion. Laboratory studies revealed a WBC count of 14,400 cells $/ \mathrm{mm} 3$, ESR of $15 \mathrm{~mm} / \mathrm{hr}$, and CRP of $3.4 \mathrm{mg} / \mathrm{dL}$. The patient was suspected of having septic arthritis and underwent a fluoroscopically guided aspiration of the right hip which yielded no fluid. Subsequently, the patient received an MRI of the pelvis which revealed enhancement of the gluteal muscles consistent with pyomyositis (Figure 2). The patient underwent a CT guided muscle biopsy and culture; these cultures demonstrated no growth. The patient was empirically treated with clindamycin for three weeks. Her symptoms promptly resolved, and by three weeks she had resumed her normal activities and showed no signs of infection.

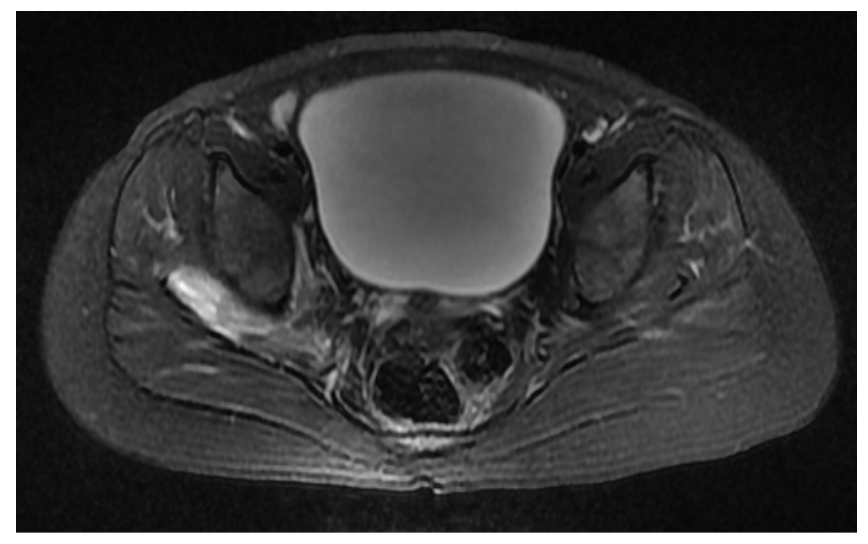

FIGURE 2: MRI of the pelvis of a 3 year old female demonstrating abnormal enhancement of the right gluteal muscles indicative of pyomyositis (axial T2). 


\section{DISCUSSION}

Primary pyomyositis is bacterial infection of skeletal muscle that most commonly affects children. ${ }^{3}$ Historically, pyomyositis was considered a disease of the tropics; in temperate climates it is estimated to account for only 1 in 4000 pediatric admissions. ${ }^{4}$ Primary pyomyositis typically involves the quadriceps (26.3\%), iliopsoas (14.0\%) and gluteal muscles (10.8\%); approximately $77 \%$ of cases involve Staphylococcus aureus. ${ }^{3,5}$ Patients present with pain, swelling, fever, and elevated inflammatory markers. If left untreated, pyomyositis can lead to abscess formation and sepsis. Diagnosis often depends on MRI, which has a high sensitivity for detecting muscle edema and abscess formation. ${ }^{3}$ If diagnosed early, pyomyositis can be treated with antibiotics alone; however, if an abscess has formed, drainage must be performed either by an open procedure or $\mathrm{CT} /$ ultrasound guidance. $^{3}$

Due to the nearly identical presentation of septic arthritis and pyomyositis, some authors have advocated for the routine use of MRI in children suspected of having septic arthritis. ${ }^{6,7}$ A single center prospective study compared the clinical and laboratory findings of 25 pediatric patients with septic hip arthritis or pericapsular pyomyositis. ${ }^{6}$ The investigators found no significant difference in the Kocher criteria or CRP between patients with septic arthritis and pyomyositis. Oddly, pyomyositis was encountered nearly twice as often as septic arthritis during the two year study period. The authors concluded that MRI was the most useful test for distinguishing the two diagnoses. They recommended FAST-sequence MRI (coronal STIR and axial T2) be performed within 2 hours prior to ultrasonography and aspiration in children with an irritable hip. ${ }^{6}$ If the MRI will take longer to obtain than two hours, a delay in ultrasonic guided hip aspiration is not recommended.

To further complicate matters, pyomyositis has been reported to occur alongside septic arthritis. ${ }^{7} \mathrm{~A}$ retrospective review of 87 children who were treated for septic arthritis found that adjacent infections such as pyomyositis or osteomyelitis were found on MRI in $59 \%$ of cases.7 Having three or more risk factors such as CRP $>1.38 \mathrm{mg} / \mathrm{dL}$, duration of symptoms $>3$ days, platelets $<314,000$ cells $/ \mathrm{mm}^{3}$, or a WBC count $>8,600$ cells $/ \mathrm{mm} 3$ was associated with an $80 \%$ positive predictive value for having adjacent infections; the review concluded that such patients may benefit from preoperative MRI to guide surgical management. ${ }^{7}$

The authors agree that MRI can be a valuable study to guide diagnosis and management in children with suspected septic hip arthritis. However, whether this study should be ordered routinely or prior to joint aspiration is less clear; the authors question the cost effectiveness and practicality of doing so. In many centers, MRI is unavailable on an emergent basis, and many young patients require anesthesia to successfully undergo the study. Performing an MRI to identify alternative infectious processes has the potential to delay the management of septic arthritis. The cases presented here represent the surgeons' first encounters with negative hip aspirate cultures with pyomyositis mimicking septic arthritis. Were pyomyositis seen more commonly, the diagnostic yield of an initial MRI would greatly increase. Pyomyositis is more common in warmer climates and in the summer months; therefore, the most appropriate clinical protocol may depend on the local incidence of these illnesses. At Cabell Huntington Hospital in WV, the current protocol is to perform an MRI after negative synovial fluid analysis ( $<50,000$ cells $/ \mathrm{mm}^{3}$, negative Gram stain). This protocol is being re-assessed with adjunctive MRI recommended due to $59 \%$ of septic arthritis patients having adjacent infections of pyomyositis or osteomyelitis.

\section{CONCLUSION}

This report presents two pediatric cases in which pyomyositis was initially mistaken for septic arthritis of the hip, resulting in a delay in management and an unnecessary arthrotomy in one patient. In both cases, the hip aspirate and cultures were negative and the correct diagnosis was revealed using MRI. $\mathrm{MRI}$ is a valuable tool that can effectively distinguish between septic arthritis and pyomyositis. It will guide the management especially in questionable or complicated cases where synovial fluid aspiration is unable to be obtained promptly. MRI is also useful in identifying adjacent spread of infection in patients with septic arthritis that may otherwise go mds.marshall.edu/mjm

(c) 2022 Marshall Journal of Medicine
Marshall Journal of Medicine Volume 4 Issue 2 
undiagnosed.

\section{AUTHOR AFFILIATIONS}

1. Marshall University Joan C. Edwards School of Medicine, Huntington, West Virginia

\section{REFERENCES}

1. Kocher MS, Zurakowski D, Kasser JR. Differentiating between septic arthritis and transient synovitis of the hip in children: an evidence-based clinical prediction algorithm. The Journal of Bone and Joint Surgery American volume. 1999;81(12):1662-70.

2. Singhal R, Perry DC, Khan FN, Cohen D, Stevenson $H L$, James $L A$, et al. The use of CRP within a clinical prediction algorithm for the differentiation of septic arthritis and transient synovitis in children. The Journal of Bone and Joint Surgery British volume. 2011;93(11):155661.

3. Bickels J, Ben-Sira L, Kessler A, Wientroub S. Primary pyomyositis. The Journal of Bone and Joint Surgery American volume. 2002;84$a(12): 2277-86$.

4. Gubbay AJ, Isaacs D. Pyomyositis in children. The Pediatric Infectious Disease Journal. 2000;19(10):1009-12; quiz 13.

5. Chiedozi LC. Pyomyositis. Review of 205 cases in 112 patients. American Journal of Surgery. 1979;137(2):255-9.

6. Mignemi ME, Menge TJ, Cole HA, Mencio GA, Martus JE, Lovejoy S, et al. Epidemiology, diagnosis, and treatment of pericapsular pyomyositis of the hip in children. Journal of Pediatric Orthopedics. 2014;34(3):316-25.

7. Rosenfeld S, Bernstein DT, Daram S, Dawson J, Zhang W. Predicting the presence of adjacent infections in septic arthritis in children. Journal of Pediatric Orthopedics. 2016;36(1):70-4. 